\title{
Geometria analítica com GeoGebra usando a Teoria dos Campos Conceituais de Vergnaud no ensino médio
}

Candido dos Santos Silva*, Miguel Raymundo Flores Santibanez ${ }^{* *}$, Delfa Mercedes Huatuco Zuasnábar ${ }^{\star \star \star}$

\section{Resumo}

Os docentes estão inserindo a utilização da tecnologia para incrementar e dinamizar suas aulas, de modo a prender a atenção dos estudantes, possibilitando a interação entre a teoria e a prática em sala de aula. Este trabalho apresenta os resultados obtidos de uma pesquisa quali-quantitativa de trabalho de campo utilizando o software GeoGebra e suas funcionalidades nas aulas de geometria analítica: ponto e reta, tendo como base as atividades propostas para uma turma de ensino médio. Destaca-se o uso da Teoria dos Campos Conceituais de Vergnaud e do software no auxílio ao desenvolvimento do processo educacional, proporcionando novos horizontes, apoiando os processos de ensino e aprendizagem dos estudantes.

Palavras-chave: GeoGebra. Ensino de geometria analítica. Teoria dos Campos Conceituais.

\section{Introdução}

O ensino de matemática, em particular de geometria analítica usando ferramentas como softwares educativos, por exemplo, o GeoGebra, está de acordo com os Parâmetros Curriculares Nacionais (BRASIL, 2010), que têm o intuito de melhorar o processo de ensino-aprendizagem, motivando mais os estudantes e tornando as aulas cada vez mais lúdicas, dinâmicas e interativas.

A metodologia usada no desenvolvimento das atividades dos estudantes foi a Teoria dos Campos Conceituais, com o objetivo de dar destaque ao conteúdo do conhecimento. Esta teoria afirma

* Licenciado em Informática, Secretaria Estadual de Educação de Roraima. E-mail: candidossilva@gmail.com

** Mestre em Ciência da Computação, chefe do Departamento de Ciência da Computação da Universidade Federal de Roraima. E-mail: miguel.santibanez@gmail.com

*** Mestre em Ciência da Computação, coordenador do curso de Ciência da Computação da Universidade Federal de Roraima. E-mail: delfa.zuasnabar@ufrr.br 
[...] que uma abordagem mais frutífera, para o desenvolvimento cognitivo das crianças, seria promovida pela utilização de uma estrutura que se refere aos conteúdos do conhecimento em si próprio e à análise conceitual do domínio (VERGNAUD; GUERSHON; CONFREY, 1994, tradução nossa).

Este trabalho apresenta o resultado de uma pesquisa desenvolvida para o $3^{\circ}$ ano do ensino médio e realizada numa escola estadual com a participação de uma turma de 25 estudantes. $O$ interesse no tema surgiu após a observação, nas aulas de matemática, de que os estudantes não conseguem associar a teoria e a prática no que se refere aos conteúdos de geometria analítica.

O problema de pesquisa proposto foi: de que forma o software GeoGebra pode ser utilizado como ferramenta pedagógica para apoiar a aprendizagem do conteúdo de geometria analítica, baseando-se na Teoria dos Campos Conceituais, de Vergnaud? O objetivo deste trabalho foi analisar a contribuição do GeoGebra no ensino de conceitos da geometria analítica: ponto e reta, tendo como base as atividades propostas.

\section{Fundamentação teórica}

Esta seção aborda os assuntos que dão suporte a esta pesquisa: geometria analítica, GeoGebra e Teoria dos Campos Conceituais, de Vergnaud.

\section{Geometria analítica}

A geometria analítica tem, entre suas características, o estabelecimento de conexões entre geometria e álgebra, pois, por exemplo, permite compreender as soluções de um sistema linear de duas incógnitas por meio de retas em um plano ou, então, representar, por meio de uma equação, uma figura bidimensional ou tridimensional. Assim, o século XVII foi marcado por um grande avanço na área matemática, ao ser esta desligada da simples aplicação às necessidades econômicas e tecnológicas. Dante entende o estudo da geometria analítica

[...] por seus elementos primitivos, o ponto e a reta, observando como o recurso de processos algébricos imprime uma precisão nas medidas e nos cálculos não encontrada na Geometria e como, por outro lado, a representação geométrica torna concreta as expressões algébricas na maioria das vezes tão abstratas (2013, p. 29).

As propriedades geométricas das curvas foram "traduzidas" assim por meio de equações, e os resultados da álgebra foram interpretados geometricamente. Dessa forma, é sensato concordar com a maioria dos historiadores que considera as decisivas contribuições dos matemáticos franceses Descartes e Fermat, no século XVII, como a origem essencial da matéria, pelo menos em seu espírito moderno. Só após as contribuições desses dois matemáticos encontramos a geometria analítica sob a forma como a conhe- 
cemos. De qualquer modo, para que a geometria analítica pudesse assumir sua apresentação atual, altamente prática, teve que aguardar o desenvolvimento do simbolismo algébrico.

No estudo de geometria analítica, ponto e reta, algumas definições são importantes, como: sistema cartesiano ortogonal, distância entre dois pontos, coordenadas do ponto médio de um segmento de reta, inclinação de uma reta, equação da reta, posições relativas de duas retas no plano (retas paralelas, retas concorrentes, intersecção de duas retas), perpendicularidade de duas retas e área de uma região triangular.

$\mathrm{Na}$ sequência, apresentamos algumas definições de geometria analítica, segundo Dante (2013):

- sistema cartesiano ortogonal: são retas orientadas que permitem a localização de pontos no plano ou no espaço;

- distância entre dois pontos: é o comprimento do segmento de reta entre dois pontos iguais e diferentes;

- coordenadas do ponto médio de um segmento de reta: corresponde ao ponto médio de abscissa $\mathrm{x}$ e ordenada y de um segmento de reta;

- inclinação de uma reta: é o ângulo determinado entre o eixo e uma reta qualquer no sentido anti-horário;
- equação da reta: a partir de um ponto $\mathrm{P}(\mathrm{x}, \mathrm{y})$ e de um declividade $\mathrm{m}$, determina-se a equação da reta;

- posições relativas de duas retas no plano (retas paralelas, retas concorrentes, intersecção de duas retas): são paralelas quando têm inclinações iguais; concorrentes, quando têm inclinações diferentes; e há intersecção quando têm um ponto em comum, satisfazendo simultaneamente as equações dessas duas retas;

- perpendicularidade de duas retas: a intersecção entre duas retas determina um ângulo de $90^{\circ}$;

- área de uma região triangular: é o valor numérico atribuído a uma região delimitada por três pontos distintos, contidos numa mesma reta dois a dois.

Postulado da determinação da reta são dois pontos distintos que determinam uma única (uma, e uma só) reta que passa por eles (DOLCE; POMPEO, 2013).

Os pontos A e B distintos determinam uma reta que indicamos por $\vec{A} \vec{B}$.

$$
\underset{\mathrm{A}}{(\mathrm{A} \neq \underset{\mathrm{A}}{\mathrm{B}}, \underset{\mathrm{B}}{\mathrm{B}} \mathrm{A} \mathrm{r}, \mathrm{B} \underset{\mathrm{B}}{\mathrm{c}} \mathrm{r})} \Rightarrow \mathrm{r}=\overrightarrow{\mathrm{AB}} .
$$

A expressão "duas retas coincidentes” é equivalente a uma única reta. Este postulado é de grande importância na geometria analítica, pois serve de base para demonstrações mais complexas. 
A origem da geometria analítica é uma ideia muito simples, introduzida por Descartes no século XVII, mas extremamente original. É a criação de um sistema de coordenadas que identifica um ponto $(\mathrm{P})$ do plano com um par de números reais $(\mathrm{x}, \mathrm{y})$. Partindo disso, podemos caracterizá-la como:

a) o estudo das propriedades geométricas de uma figura com base em uma equação (nesse caso, são as figuras geométricas que estão sob o olhar da álgebra);

b) o estudo dos pares ordenados de números $(\mathrm{x} ; \mathrm{y})$ que são soluções de uma equação, por meio das propriedades de uma figura geo- métrica (nesse caso, é a álgebra que está sob o olhar da geometria) (SECRETARIA DE EDUCAÇÃO BÁSICA, 2006).

Percebe-se que o trabalho com a geometria analítica permite a articulação entre geometria e álgebra. Assim, para que essa articulação seja significativa para o estudante, o professor deve trabalhar o entendimento de figuras geométricas por meio de equações, e o entendimento de equações, por meio de figuras geométricas.

O Quadro 1 apresenta um resumo do conteúdo e da habilidade descritos por Dante (2013), que os estudantes devem adquirir durante o processo.

Quadro 1 - Conteúdo e habilidade baseados em objetos de conhecimento

\begin{tabular}{|c|c|c|}
\hline $\begin{array}{c}\text { Conteúdo } \\
\text { Geometria analítica: ponto e reta }\end{array}$ & $\begin{array}{c}\text { Objetos de conhecimento } \\
\text { (Associados às Matrizes de } \\
\text { Referência para o Enem 2009) }\end{array}$ & Habilidade \\
\hline Sistema cartesiano ortogonal & \multirow{5}{*}{$\begin{array}{l}\text { Conhecimentos } \\
\text { algébricos/geométricos - plano } \\
\text { cartesiano, retas }\end{array}$} & \multirow{8}{*}{$\begin{array}{l}\text { - Identificar características de } \\
\text { figuras planas ou espaciais. } \\
\text { - Resolver situação-problema que } \\
\text { envolva conhecimentos } \\
\text { geométricos de espaço e forma. } \\
\text { - Identificar representações } \\
\text { algébricas que expressem a relação } \\
\text { entre grandezas. } \\
\text { - Interpretar gráfico cartesiano que } \\
\text { represente relações entre } \\
\text { grandezas. Resolver situação-problema cuja } \\
\text { modelagem envolva } \\
\text { conhecimentos algébricos. }\end{array}$} \\
\hline Distância entre dois pontos & & \\
\hline $\begin{array}{l}\text { Coordenadas do ponto médio de } \\
\text { um segmento de reta }\end{array}$ & & \\
\hline Inclinação de uma reta & & \\
\hline Equação da r & & \\
\hline $\begin{array}{l}\text { Posições relativas de duas retas } \\
\text { no plano (retas paralelas, retas } \\
\text { concorrentes, intersecção de } \\
\text { duas retas) }\end{array}$ & \multirow{3}{*}{$\begin{array}{c}\text { Conhecimentos } \\
\text { algébricos/geométrico - par e } \\
\text { perpendicularidade }\end{array}$} & \\
\hline Perpendicularidade de duas retas & & \\
\hline Área de uma região t & & \\
\hline
\end{tabular}

Fonte: Dante (2013, p. 35). 
Na literatura, encontramos diversos softwares para o ensino de geometria analítica disponíveis, podemos citar: Cabri Geometry, Dr Geo, Euklid, Geoplan, Régua e Compasso, Graphamática, S-logo e GeoGebra.

\section{GeoGebra}

Na realização desta pesquisa, foi utilizado o software GeoGebra por ser livre, gratuito, escrito em linguagem Java, compatível com diferentes sistemas operacionais, leve, fácil de instalar e de manusear, voltado para a aprendizagem e o ensino em todos os níveis, possibilitando que se trabalhe geometria interativa, álgebra, cálculo e estatística (GEOGEBRA, 2017).

Uma das vantagens de se utilizar o software GeoGebra como ferramenta pedagógica é a sua capacidade de proporcionar dupla correspondência dos objetos, ou seja, cada expressão disposta na janela algébrica corresponde a um objeto na janela gráfica e vice-versa. Com isso, o estudante dispõe da possibilidade de visualizar os cálculos que estão sendo realizados, facilitando a compreensão do conteúdo trabalhado (HOHENWARTER, 2015).

Foi por meio da utilização, durante as aulas, do software GeoGebra, que possibilita interação, que os estudantes adquiriram uma visão diferenciada da geometria analítica, alcançando as habilidades e competências descritas no Quadro 1.
Teoria dos Campos Conceituais, de Vergnaud

A Teoria dos Campos Conceituais, de Gérard Vergnaud, é uma teoria cognitivista neopiagetiana do processo de conceitualização do real, que tem por objetivo oferecer uma estrutura e alguns princípios básicos às pesquisas sobre atividades cognitivas, principalmente àquelas que dependem da ciência e da técnica,

[...] mas não é específica da matemática, embora, inicialmente, tenha sido elaborada para explicar o processo de conceitualização progressiva das estruturas aditivas, das estruturas multiplicativas, das relações número-espaço e da álgebra (VERGNAUD, 1993, p. 21).

Essa teoria, apesar de proporcionar uma estrutura à aprendizagem e envolver a didática, não é uma teoria didática.

A principal finalidade é

[...] propor uma estrutura que permita compreender as filiações e rupturas entre conhecimentos, do ponto de vista de seu conteúdo conceitual (VERGNAUD, 1993, p. 62).

O campo conceitual é definido por Vergnaud como

[...] um conjunto informal e heterogêneo de problemas, situações, conceitos, relações, estruturas, conteúdos e operações de pensamento, conectados uns aos outros e, provavelmente, entrelaçados durante o processo de aquisição (1982, p. 45, tradução nossa).

De acordo com o autor, três argumentos principais levaram à construção do conceito de campo conceitual 
(VERGNAUD, 1983). O primeiro: um conceito não pode se formar a partir de um único tipo de situação. O segundo: é necessário mais de um conceito para se analisar uma única situação. E o terceiro: a construção e a apropriação de todas as propriedades em um conceito são um processo que requer um tempo para entender, podendo gerar analogias e mal-entendidos entre situações, concepções, procedimentos ou significantes.

Segundo Moreira (2002), os interesses dos estudos de Gérard Vergnaud estão voltados para dois principais campos conceituais: $o$ das estruturas aditivas e $o$ das estruturas multiplicativas. O campo conceitual das estruturas aditivas é o conjunto de situações cujo domínio requer uma adição, uma subtração ou uma combinação de tais operações. O campo conceitual das estruturas multiplicativas consiste em todas as situações que podem ser analisadas como problemas, sejam de proporções simples ou múltiplas, em que é necessária uma multiplicação, uma divisão ou uma combinação dessas operações.

Apesar de a definição de campo conceitual ser bem clara, a linha de fronteira cognitiva entre campos conceituais não está necessariamente bem definida. A principal razão para isso é que há uma ruptura no conhecimento humano. Por exemplo, há uma filiação entre as estruturas aditivas e multiplicativas. Apesar disso, há especificidade suficiente nos problemas cognitivos gerados pelas estruturas aditivas, de um lado, e pelas estruturas multiplicativas, de outro, o que nos permite estudar esses dois campos conceituais separadamente (VERGNAUD, 1988).

Vergnaud (1983, 1988) entende que, para estudar e compreender como os conceitos evoluem na mente de um sujeito por meio de suas experiências, é preciso considerar o conceito (C) como uma terna de conjuntos, ou seja, $\mathrm{C}=(\mathrm{S}, \mathrm{I}, \mathrm{R})$, em que:

- S é o conjunto de situações que dão significado e utilidade ao conceito;

- I é o conjunto de invariantes operatórios associados ao conceito (objetos, propriedades e relações), que pode ser reconhecido e usado pelos sujeitos de forma a analisar e dominar aquelas situações;

- $\mathrm{R}$ é o conjunto de significantes, isto é, a representação simbólica, linguística, gráfica ou gestual, que pode ser utilizado para representar aqueles invariantes e, dessa forma, representar as situações e os procedimentos para lidar com eles.

Em termos psicológicos, o autor explicita que: S, o referente, é a realidade; e I e $\mathrm{R}$ representam os dois aspectos integrantes do pensamento, o significado (I) e o significante (R).

É fundamental considerar esses três conjuntos simultaneamente - situações, invariantes operatórios e representações simbólicas - ao longo da aprendizagem, para estudar o desenvolvimento e o uso de um conceito (VERGNAUD, 1983). 
Um conceito não é uma mera definição, refere-se a um conjunto de situações envolvidas em um conjunto de diferentes invariantes operatórios; e suas propriedades podem ser expressas por meio de diferentes representações linguísticas e simbólicas (VERGNAUD, 1998).

\section{Metodologia}

A abordagem da pesquisa tem naturezas qualitativa e quantitativa e apresenta envolvimento de dados estatísticos. De acordo com Goodenough, "uma das características da pesquisa híbrida é a de possibilitar um processo de continuidade e interação, sendo compartilhada por todos a responsabilidade pela execução e pelo sucesso dos resultados" (1971, p. 23, tradução nossa).

Segundo Bogdan e Biklen:

Os estudos que empregam uma metodologia qualitativa podem descrever a complexidade de determinado problema, analisar a interação de certas variáveis, compreender e classificar processos dinâmicos vividos por grupos sociais.

A abordagem da investigação qualitativa exige que o mundo seja examinado com a ideia de que nada é trivial, que tudo tem potencial para constituir uma pista que nos permita estabelecer uma compreensão mais esclarecedora do nosso objeto de estudo (BOGDAN; BIKLEN, 1994, p. 32).

Já a abordagem quantitativa geralmente implica a utilização de questionários e o envolvimento estatístico para a análise dos dados. De acordo com Prodanov e Freitas, "tudo pode ser quantificável, o que significa traduzir em números opiniões e informações para classificá-las e analisá-las” (2013, p. 23). No desenvolvimento da pesquisa de natureza quantitativa, devemos formular hipóteses e classificar a relação entre as variáveis, para garantir a precisão dos resultados, evitando contradições nos processos de análise e interpretação e utilizando recursos e técnicas estatísticas (percentagem).

Como trabalha-se com a utilização de software matemático, a pesquisa tem aspecto dedutivo na modalidade pesquisa de campo, também relacionando atividades propostas. $\mathrm{O}$ método dedutivo visa a explicar os passos e procedimentos realizados pelos estudantes durante a resolução das atividades propostas, analisando os questionários e deduzindo as informações transcritas nos instrumentos. Para Gil, a pesquisa dedutiva "parte de princípios reconhecidos como verdadeiros e indiscutíveis e possibilita chegar a conclusões de maneira puramente formal, isto é, em virtude unicamente de sua lógica" (2008, p. 12).

$\mathrm{O}$ universo da pesquisa baseia-se nas turmas regulares do $3^{\circ}$ ano do ensino médio de uma escola estadual. As aulas foram divididas em quatro etapas, em que foram definidos os objetivos e metas a serem alcançados. Nas aulas 1 e 2, foram trabalhados conteúdos de geometria analítica, ponto e reta, em que problemas 
foram resolvidos, e foi mostrado como esses problemas podem ser representados/resolvidos com o auxílio do software GeoGebra. Após essa ambientação com o software, foi apresentada a Teoria dos Campos Conceituais, de Vergnaud.

$\mathrm{Na}$ aula 3, propuseram-se situações-problema, para que os estudantes utilizassem o software para resolução das atividades propostas aplicando a Teoria dos Campos Conceituais, de Vergnaud. Finalmente, na aula 4, foram aplicados questionários aos estudantes e ao professor colaborador, visando a obter informações sobre a aplicabilidade do software e vantagens e desvantagens na sua utilização.

\section{Análise dos resultados}

Para a análise dos resultados, foi realizada uma tabulação qualitativa e quantitativa dos dados das atividades propostas aos estudantes, na qual estão descritas as respostas obtidas na manipulação do software GeoGebra. Na aula 4, foram aplicados questionários aos estudantes, com o objetivo de verificar a eficácia da metodologia utilizando o software GeoGebra e a Teoria dos Campos Conceituais, de Vergnaud, como ferramentas pedagógicas, instigando sobre 0 conteúdo, sobre pontos positivos e negativos ao trabalhar com essa metodologia.

A seguir, representamos graficamente (Gráfico 1) a resposta a uma das perguntas do questionário nos quesitos "sim", "não" e "um pouco".

Gráfico 1 - Respostas dos alunos a uma questão do questionário

Questão:o GeoGebra ajudou-oa ver a matemática sobre outra ótica?

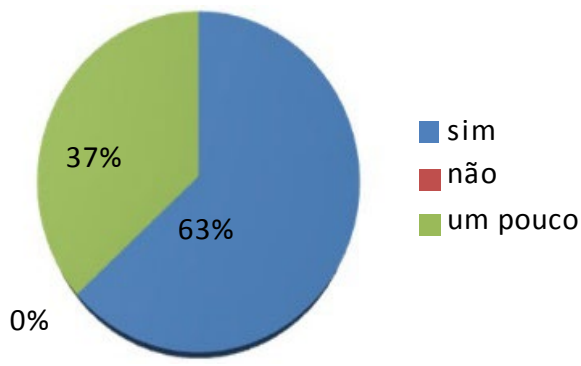

Fonte: elaboração dos autores.

No Quadro 2, são descritas as justificativas às respostas "sim".

Quadro 2 - Resposta "sim" dos alunos e suas justificativas

\begin{tabular}{|l|l|}
\hline Aluno & \multicolumn{1}{|c|}{ Justificativa à resposta } \\
\hline I-01 & $\begin{array}{l}\text { "Usando o computador é completamente } \\
\text { diferente." }\end{array}$ \\
\hline I-02 & "Porque vejo a matemática melhor." \\
\hline I-07 & $\begin{array}{l}\text { "Pois facilitou a compreensão sobre deter- } \\
\text { minados assuntos." }\end{array}$ \\
\hline I-08 & "Muito mais fácil entender o gráfico." \\
\hline I-09 & "O programa é bom, facilita os cálculos." \\
\hline I-10 & "Pois facilitou a compreensão da disciplina." \\
\hline I-11 & $\begin{array}{l}\text { "Porque é uma maneira mais fácil de resol- } \\
\text { ver as contas." }\end{array}$ \\
\hline I-14 & "Antes eu não entendia bem, agora sei." \\
\hline I-15 & $\begin{array}{l}\text { "Uso de algumas ferramentas clareou a mi- } \\
\text { nha mente." }\end{array}$ \\
\hline I-17 & "Porque facilita a aprender o assunto." \\
\hline I-18 & $\begin{array}{l}\text { "Porque nesse conteúdo não precisamos } \\
\text { ficar fazendo tudo a mão." }\end{array}$ \\
\hline I-19
\end{tabular}

Fonte: elaboração dos autores. 
No Quadro 3, são descritas as justificativas às respostas "não".

Quadro 3 - Resposta "não" dos alunos e suas justificativas

\begin{tabular}{|l|l|}
\hline Aluno & Justificativa à resposta \\
\hline Não houve marcação no quesito não. \\
\hline
\end{tabular}

Fonte: elaboração dos autores.

No Quadro 4, são descritas as justificativas às respostas "um pouco".

Quadro 4 - Resposta "um pouco" dos alunos e suas justificativas

\begin{tabular}{|l|l|}
\hline Aluno & Justificativa à resposta \\
\hline $\mathrm{I}-03$ & $\begin{array}{l}\text { "Porque não mostra o cálculo como } \\
\text { fazer." }\end{array}$ \\
\hline $\mathrm{I}-04$ & "Porque nos ajudou muito." \\
\hline $\mathrm{I}-05$ & $\begin{array}{l}\text { "Pois tenho dificuldade na compre- } \\
\text { ensão da disciplina." }\end{array}$ \\
\hline $\mathrm{I}-06$ & $\begin{array}{l}\text { "Sim, pela dica da informática e a } \\
\text { facilidade que podemos." }\end{array}$ \\
\hline $\mathrm{I}-12$ & $\begin{array}{l}\text { "Porque tenho dificuldade em ma- } \\
\text { temática." }\end{array}$ \\
\hline $\mathrm{I}-13$-16
\end{tabular}

Fonte: elaboração dos autores.
No Quadro 2, estão listados os doze estudantes que responderam "sim", passando a visualizar, por meio do software, a matemática sob uma nova ótica. O Quadro 3 mostra que não houve marcação no quesito "não". No Quadro 4, são listados os sete estudantes que responderam "um pouco". Sendo assim, podemos afirmar que o software como metodologia contribui para aquisição do conhecimento.

A geometria analítica requer que os discentes desenvolvam desde habilidades e competências com simples representações de pontos, figuras e relações de equação no plano cartesiano até a resolução de problemas com equações e inequações, identificação de equação de reta, circunferência e formas cônicas. Esta relação entre geometria e álgebra foi desenvolvida por René Descartes por volta do século XVII (VALERIO, 2013).

A seguir, apresentamos o Quadro 5, uma aplicação com o GeoGebra baseado na Teoria dos Campos Conceituais, de Vergnaud. Selecionamos o seguinte exemplo como referência: 
Quadro 5 - Aplicação com o GeoGebra baseado na Teoria dos Campos Conceituais

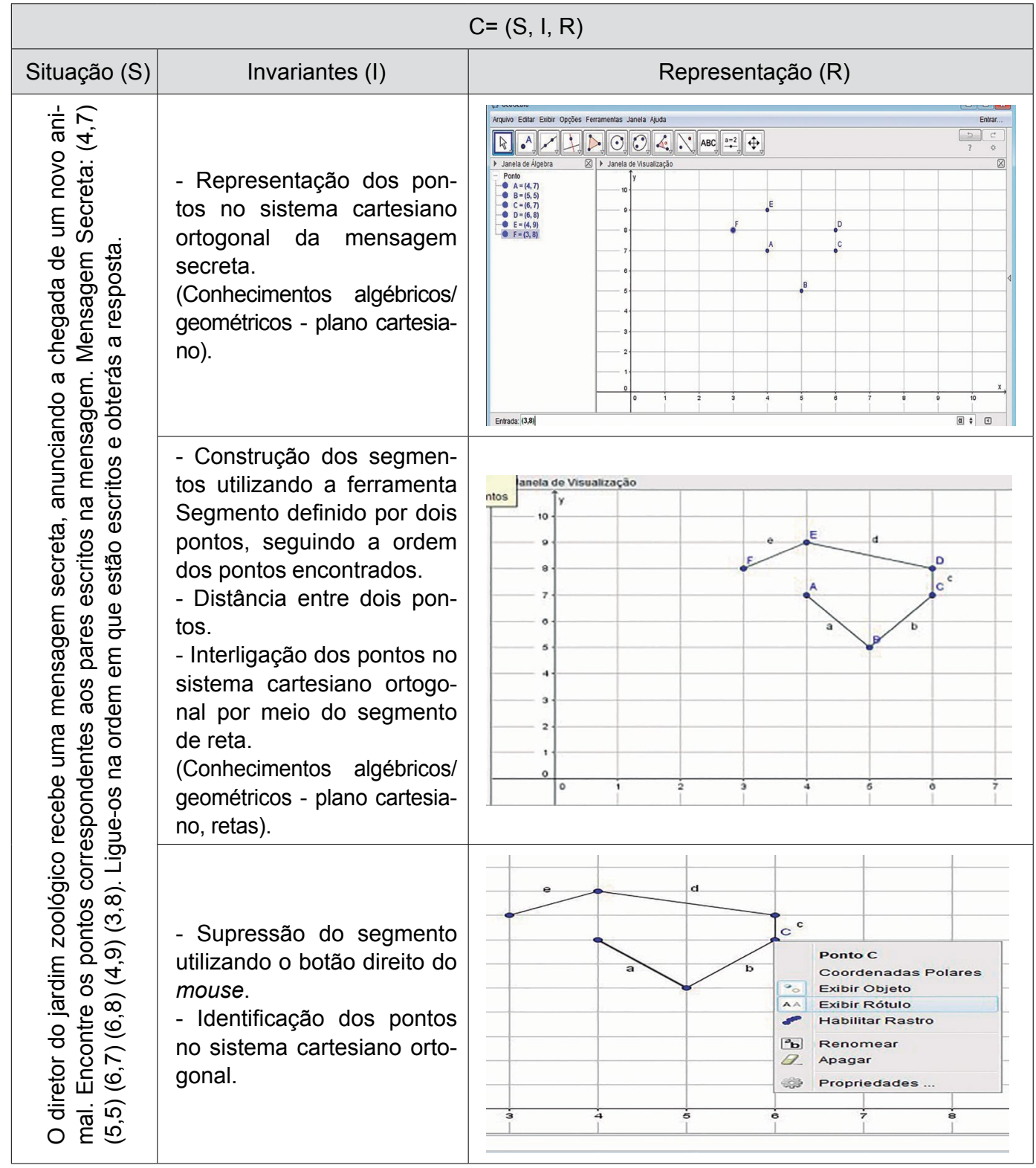

Fonte: elaboração dos autores. 


\section{Conclusões}

Este trabalho teve como objetivo analisar a contribuição do GeoGebra no ensino dos conceitos ponto e reta da geometria analítica, tendo como base as atividades propostas e a Teoria dos Campos Conceituais, de Vergnaud, dando importância aos conhecimentos prévios e/ ou adquiridos em sala pelos estudantes.

Foram realizadas uma reflexão e uma discussão a respeito da implantação e do uso de softwares dinâmicos gratuitos para ensinar matemática no ensino médio, com o intuito de construir atividades de geometria analítica que possam ser resolvidas com o auxílio do software GeoGebra, desenvolvendo uma proposta didática apoiada no uso da tecnologia.

Dessa forma, apresenta-se como resultados a contribuição do GeoGebra no desenvolvimento das atividades propostas, embasada na prática, possibilitando aos professores aulas mais dinâmicas e organizadas de maneira a despertar o "gosto" pela matemática. O estudo também visou ao desenvolvimento de novas técnicas, que serão desenvolvidas com os softwares e com a resolução dos problemas de construção geométrica.

A Teoria dos Campos Conceituais, de Vergnaud, contribuiu com a formação de conceitos; os estudantes tiveram a oportunidade de aplicar os seus conhecimentos implícitos ou explícitos nos problemas propostos, utilizando o soft- ware GeoGebra e aplicado os conteúdos de ponto e reta de geometria analítica.

As respostas aos questionários dos estudantes foram satisfatórias. Conforme apresentado na análise quantitativa, a maioria dos estudantes conseguiu desenvolver as atividades propostas utilizando o GeoGebra. Com o desenvolvimento da pesquisa, percebe-se que o uso de tecnologias está cada vez mais presente no ambiente escolar de forma direta e indireta, como: celulares, tablet, notebook, netbook e outros. Nesses ambientes, os docentes estão cada vez mais inserindo a utilização de recursos para incrementar e dinamizar suas aulas, de modo a prender atenção dos estudantes.

Apesar de ter sua origem em uma ideia relativamente simples, o estudo da geometria analítica, por parte dos estudantes, demonstra ser de difícil entendimento, apresentando grandes dificuldades, principalmente no que se refere à visualização dos conceitos envolvidos e da capacidade de localização no plano cartesiano, necessitando complementar o seu ensino com ferramentas que permitam o entendimento das figuras, associando a isso as equações e demonstrações. 


\section{Analytic geometry with GeoGebra using the Theory of the Conceptual Fields of Vergnaud in high school}

\section{Abstract}

The teaching staff are inserting the use of technology to increase and boost their classes, in a way to catch the students attention, providing the interaction between the theory and the practice inside the classroom. This work presents the results obtained in a qualitative and quantitative research of fieldwork using the GeoGebra software and its functionalities, in classes of Analytic Geometry: point and line, having as base the activities proposed to the High School class. There is also the Conceptual Fields of Vergnaud that are highlighted and the use of the software helping the development of the educational process providing new horizons, supporting the teaching and learning process of the students.

Keywords: Analytic geometry teaching. GeoGebra. Theory of the Conceptual Fields.

\section{Referências}

BOGDAN, R.; BIKLEN, S. Investigação qualitativa em educação: uma introdução à teoria e aos métodos. Porto, Portugal: Porto Editora, 1994.

BRASIL. PCN+, Ensino Médio, Orientações Educacionais Complementares aos Parâmetros Curriculares Nacionais. Ciências da Natureza, Matemática e suas Tecnologias. 2010. Disponível em: <http://portal.mec.gov.br/seb/arquivos/ pdf/CienciasNatureza.pdf $>$. Acesso em: $01 \mathrm{dez}$. 2017.

DANTE, L. R. Matemática: contexto e aplicações. 2. ed. São Paulo: Ática, 2013. v. 3.
DOLCE, O.; POMPEO, J. N. Fundamentos de Matemática Elementar - Geometria Plana. 9. ed. São Paulo: Atual, 2013.

GEOGEBRA, Site Oficial. 2017. Disponível em: <https://www.geogebra.org/>. Acesso em: 30 mar. 2017.

GIL, A. C. Métodos e técnicas de pesquisa social. 6. ed. São Paulo: Atlas, 2008.

GOODENOUGH, W. Culture, language, and society. Reading: Addison Wesley, 1971.

HOHENWARTER, M. Geogebra 4 Quickstart. Tradução para português de Portugal de Antônio Ribeiro e Jorge Geraldes. 2015. Disponível em: <http://static.geogebra.org/help/ geogebraquickstart_pt_PT.pdf $>$. Acesso em: 30 maio 2015.

MOREIRA, M. A. A Teoria dos Campos Conceituais de Vergnaud, o ensino de ciências e a pesquisa nesta área. Investigações em Ensino de Ciências. Porto Alegre, v. 7, n. 1, p. 7-29, 2002.

PRODANOV, C. C.; FREITAS, E. C. Metodologia do trabalho científico: métodos e técnicas da pesquisa e do trabalho acadêmico. 2. ed. Novo Hamburgo: Feevale, 2013.

RICHARDSON, R. J. Pesquisa Social: métodos e técnicas. 3. ed. São Paulo: Atlas, 1999.

SECRETARIA DE EDUCAÇÃO BÁSICA. Ciências da natureza, matemática e suas tecnologias. Brasília: Ministério da Educação, Secretaria de Educação Básica, 2006. (Orientações curriculares para o ensino médio; volume 2). Disponível em: <http://portal.mec.gov.br/seb/ arquivos/pdf/book_volume_02_internet.pdf $>$. Acesso em: 30 maio 2015.

VALERIO, S. Ensino da geometria analítica com o uso do software Geogebra. Revista Eletrônica de Educação e Ciência, São Paulo, v. 3, n. 1, p. 12-35, 2013.

VERGNAUD, G. A classification of cognitive tasks and operations of thought envolved in addition subtraction problems. In: CARPENTER, T.; MOSER, J.; ROMBERG, T. Addition and subtraction. A cognitive perspective. Hillsdale, N. J.: Lawrence Erlbaum, 1982. p. 39-65. 
A comprehensive theory of representation for mathematics education. Journal of Mathematical Behavioral, Boston, v. S17, n. 2, p. 167-181, 1998.

Multiplicative structures. In: HIEBERT, H.; BEHR, M. (Ed.). Research Agenda Mathematics Education. Number Concepts and Operations in the Middle Grades. Hillsdale, N. J.: Lawrence Erlbaum, 1988. p. 45-112.

Quelques problèmes théoriques de la didactique a propos d'um exemple: les structures additives. Atelier Internacional d'Eté: Récherche em Didactique de la Phyque. La Londe les Maures, França, 1983.

Teoria dos campos conceituais. In: NASSER, L. (Ed.). Anais do $1^{\circ}$ Seminário Internacional de Educação Matemática. Rio de Janeiro, 1993. p. 1-26.

VERGNAUD, G.; GUERSHON, H.; CONFREY, J. The Development of Multiplicative Reasoning in the Learning of mathematics. New York: State Universty of New York Press, 1994. p. 41-59. 